\title{
CRACK IDENTIFICATION IN PLATES USING 1-D DISCRETE WAVELET TRANSFORM
}

\author{
Anna Knitter-Piątkowska, Michą Guminiak \\ Poznan University of Technology, Poznań, Poland \\ e-mail: anna.knitter-piatkowska@put.poznan.pl; michal.guminiak@put.poznan.pl \\ George Hloupis \\ Technological Educational Institute of Athens, Athens, Greece \\ e-mail: hloupis@teiath.gr
}

\begin{abstract}
In the present work, the defect detection while using Discrete Wavelet Transform in rectangular plate structures is investigated. The plate bending is described by using the Boundary Element Method with boundary integral equations formulated in a modified simplified approach. The boundary elements of a constant type in a non-singular approach are implemented. Defects are introduced by additional edges forming slots or holes in relation to the basic plate domain. Estimation of the defect position is performed while using wavelet coefficients of curvature and deformation signals as well as a newly proposed moving variance estimator.

Keywords: damage detection, crack, plates, wavelet transform, Boundary Element Method
\end{abstract}

\section{Introduction}

Damage generally can be defined as a change in the material which impairs functioning of the structural element at a given moment or in the future. As a result, it may lead to destruction of the element or, in the worst case, even of the whole structure. Defects usually have the form of delaminations, cracks, local material damage due to corrosion or fatigue; they may also take the form of voids or undesired inclusions. The issue of early detection, locating and quantifying structural damage is one of the most important engineering problems because it is closely linked to safety and durability of the object.

For years, many researchers have developed different methods of identifying defects with a focus on non-destructive testing (NDT). The essence of NDT is to locate failure in the structural element without changing its properties and functionality. Further analysis and subsequent stages of identification include: classification and severity assessment of damage, determination of its position, forecasting of probability of an element or the whole structure destruction and estimating the remaining potential lifetime of the structure.

Starting from the simplest NDT technique, namely visual inspection, one can mention methods basing on information from e.g. acoustic emission (Rogers, 2005), X-rays (Shinoba et al., 2004), eddy current (Gros, 1995), ultrasonography (Zhang et al., 2004), magnetic field (Lee et al., 2004) or soft computing methods such as artificial neural networks (Waszczyszyn and Ziemiański, 2001) and evolutionary algorithms (Burczyński et al., 2004). The traditional approach, based on the analysis of natural frequencies (Dems and Mróz, 2001) and modal shapes of structure vibrations (Ostachowicz and Kaczmarczyk, 2001), is still used and further developed. However, the global static or dynamic structural response is rather insensitive to localized damage.

A promising tool in structural identification is Wavelet Transform (WT) which can surprisingly well extract the desired detailed information from numerous data representing the global response of a defective structure. The application of WT for identification of cracks in structures 
received a great attention over the last decade. Douka et al. (2003), Quek et al. (2001), Gentile and Messina (2003), Garstecki et al. (2004) as well as Kim and Melhem (2004) used the WT for crack detection in beams. The effectiveness of the discrete wavelet transform (DWT) combined with the inverse analysis for damage identification in beams was discussed in Knitter-Piatkowska and Garbowski (2013). In plate structures, a 2D WT used by Loutridis et al. (2005) and Rucka and Wilde (2004); Douka et al. (2004) used 1D WT to analytically determined mode shapes along several perpendicular lines while Chang and Chen (2004) used spatial wavelet analysis to estimate the crack size. Damage detection while using 1D and 2D DWT of a temperature field recorded on the surface of a plate structure was discussed in Ziopaja et al. (2011). More complex structures such as frames were analysed by Ovanesova and Suarez (2004) and trusses by Knitter-Piątkowska et al. (2014). The previous studies clearly demonstrate that the use of WT in search for structural damage is a promising and developing field of investigation.

Our intention in the present work is to examine performance of DWT as a method for crack identification in plates. The attention is focused on defects propagated from the external edge of a plate, which contributes new elements to the established knowledge. The position of the crack is determined as the absolute maximum of a moving variance estimator which uses the wavelet transformed response signals as the input. The feasibility of the proposed method is demonstrated by means of numerical examples. The effect of added noise on the performance of the method is investigated via noise immunity tests. The contribution of the current study is based on a simple fact which provides accurate results (even with high portions of added noise) and its implementation could be computationally efficient.

\section{The discrete wavelet transform - theoretical foundations}

In the current study, the WT will be implemented in which, for the representation of a signal $f(t)$, a linear combination of wavelet functions is applied. The theory of the WT has been presented in many publications, e.g. Meyer (1992) and Daubechies (1992). The foundations of the wavelet transformation will be mentioned below. As a matter of fact, the continuous wavelet transform of the signal $f(t)$ in the time and frequency domain can be defined as

$$
W f(a, b)=\int_{-\infty}^{\infty} f(t) \bar{\psi}_{a, b}(t) d t
$$

where the overbar denotes the complex conjugate of the function under it. The function $\psi(t)$ is called the wavelet (mother) function, it belongs to the $L^{2}(\mathcal{R})$ field and must satisfy the condition of admissibility (Mallat, 1989) which leads to the inequality

$$
\int_{0}^{\infty} \frac{|\Psi(\omega)|^{2}}{\omega} d \omega<\infty
$$

where $\Psi(\omega)$ is the Fourier transform of $\psi(t)$, and it is defined as

$$
\Psi(\omega)=\int_{-\infty}^{\infty} \psi(t) \mathrm{e}^{-\mathrm{i} \omega t} d t
$$

In this case it becomes oscillatory because its average value is equal to zero. The character of mother function may be real or complex-valued. In the considered cases, real-valued family of wavelets is applied. The set of wavelets is obtained by scaling and translating of the function $\psi$, which leads to the relation

$$
\psi_{a, b}=\frac{1}{\sqrt{|a|}} \psi\left(\frac{t-b}{a}\right)
$$


where $t$ denotes time or a space coordinate, $a$ is the scale parameter and $b$ the translation parameter. The parameters $a$ and $b$ take real values $(a, b \in \mathcal{R})$ and additionally $a \neq 0$. The element $\sqrt{|a|}$ is the scale factor which ensures constant wavelet energy regardless of the scale. It means that $\left\|\psi_{a, b}\right\|=\|\psi\|=1$.

In the present numerical approach, the leading role will be taken by DWT. DWT requires neither integration nor explicit knowledge of the scaling and wavelet function. The family of discrete wavelet functions can be obtained on the assumption that $a=1 / 2^{j}, b=k / 2^{j}$ and substitution of them into Eq. (2.4). It leads to the following relation

$$
\psi_{j, k}(t)=2^{\frac{j}{2}} \psi\left(2^{j} t-k\right)
$$

in which $k$ and $j$ are scale and translation parameters, respectively. The meaning of these parameters can be clearly illustrated for the simplest Haar wavelet (Fig. 1).

(a)

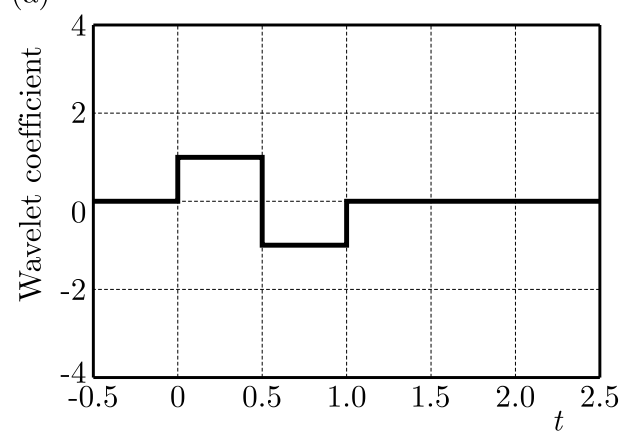

(c)

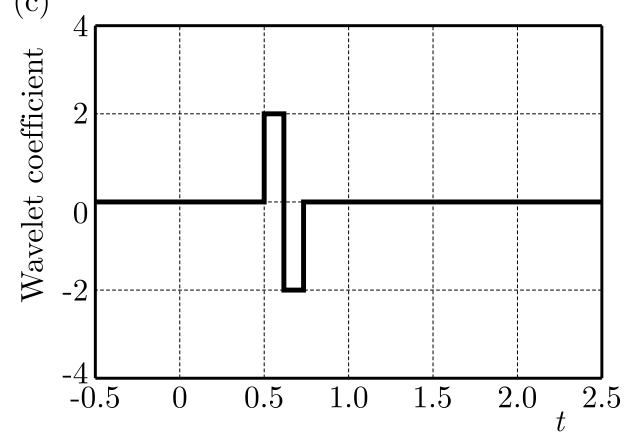

(b)

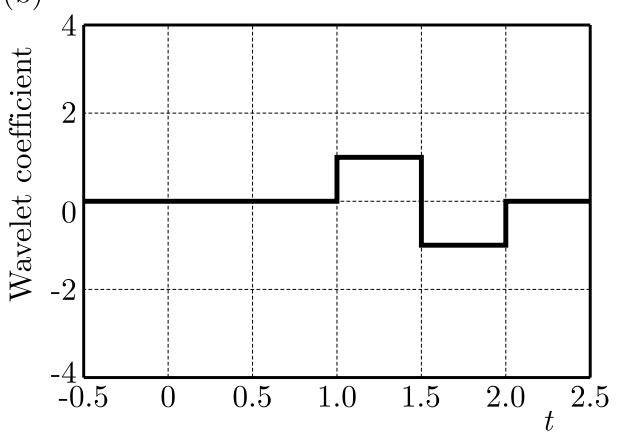

(d)

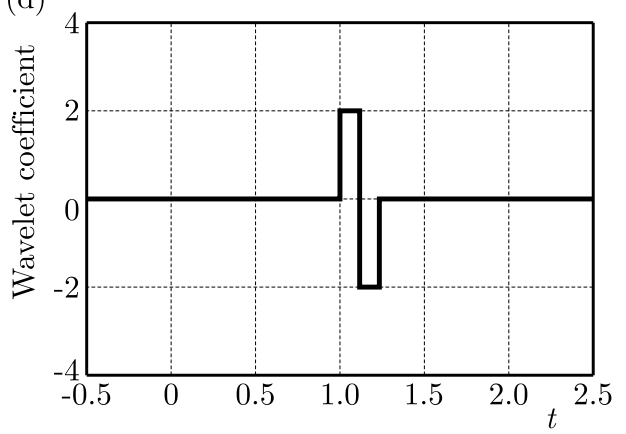

Fig. 1. Haar wavelet family: (a) mother wavelet $j=0, k=0$, (b) wavelet with parameters $j=0, k=1$, (c) $j=2, k=2$, (d) $j=2, k=4$

The main requirement (which is the one of contributions of the current study) is the use of a WT implementation algorithm that is computationally efficient in terms of required memory and processing power. The classical implementation of DWT consists of a pair of finite impulse response filters (FIR) (high-pass and low-pass) which are applied to the signal in parallel. The pyramid algorithm by Mallat (1989) computes the 1D convolution based WT at different levels of resolution. The produced coefficients are the result of a recursive convolution between the signal and corresponding filter for preselected values of $j$ and $k$. This convolution-based DWT requires a large number of arithmetic computations and memory allocations leading to quite demanding computing scheme. Towards to the direction of the computational efficient algorithmic approach, a light-weight implementation for performing the WT can be adopted. This is the Lifting Scheme (Daubechies and Sweldens, 1998; Sweldens, 1996) which requires fewer computations (half of those needed for the convolution based DWT). In general, it consists of three steps: split, lift and scale. The basic idea is to initially compute a trivial wavelet by splitting the analyzed signal into odd and even subsequences and then modifying these subsequences by consecutive predict and update steps. 
For the selection of an appropriate wavelet, we adopt general recommendations by Ovanesova and Suarez (2004) for the selection of wavelets that can perform a Fast Wavelet Transform (FWT) and satisfy symmetry and exact reconstruction. The candidate wavelets are the biorthogonal and the Haar. Fortunately, both wavelet families are supported by the Lifting Scheme. The last criterion that leads us to the choice of biorthogonal (bior.) wavelet (Cohen et al., 1992) in the current study, is that the Haar is an irregular wavelet (while the biorthogonal is not) (Meyer, 1992). This property has been proved significant for crack detection (Ovanesova and Suarez, 2004).

The biorthogonal wavelets relax the assumption of a single orthogonal basis (such as the Haar) and belong to the family of wavelets that are derived from bases that are semi-orthogonal, biorthogonal or non-orthogonal. Instead, they have primary and dual scaling $(\varphi, \widetilde{\varphi})$ and wavelet $(\psi, \widetilde{\psi})$ functions - a characteristic that provides more flexibility to the construction of wavelet base.

The scaling and wavelet are related as

$$
\int \widetilde{\psi}_{j, k}(x) \psi_{j^{\prime} k^{\prime}}(x) d x=0
$$

as soon as $j \neq j^{\prime}$ or $k \neq k^{\prime}$ and even

$$
\int \widetilde{\varphi}_{0, k}(x) \varphi_{0 k^{\prime}}(x) d x=0
$$

as soon as $k \neq k^{\prime}$.

One wavelet $(\widetilde{\psi})$ used for analysis of the signal $s$ produces coefficients $c_{j, k}$ as below

$$
\widetilde{c}_{j, k}=\int s(x) \widetilde{\psi}_{j, k}(x) d x
$$

while the other wavelet $(\psi)$ is used for synthesis of the signal $s$ from wavelet coefficients as follows

$$
s=\sum_{j, k} \widetilde{c}_{j, k} \psi_{j, k}
$$

Naming the biorthogonal wavelets follows the convention biorNr.Nd, where $\mathrm{Nr}$ is the number of the order of the wavelet or scaling functions used for signal synthesis, while $\mathrm{Nd}$ is the order of functions used for signal analysis. In our study, we adopt the bior2.2 wavelet which is presented in Fig. 2.

\section{Problem formulation and numerical analysis}

The aim of this work is to detect location of a defect provided that the defect (damage) exists in the considered plate structure. Numerical investigation is based on signal analysis of the structural static response. The plate material is assumed as linear-elastic. The plate bending is described and solved by the Boundary Element Method. The boundary integral equations are derived from a non-singular approach. Rectangular plates simply-supported along the edges are considered. The analysis of the structural response is conducted with the use of signal processing tool, namely the wavelet transformation in its discrete form. Defects in plates are modeled as slots near the plate boundary and introduced as a set of free edges. An example of the plate with a defected edge is illustrated in Fig. 3. The plate is loaded by a single concentrated $P$ force moving along the indicated line, for example 1), 2) or 3). The force $P$ can have a static or dynamic character. At the selected $D$ point measured are: deflection $w$, angle of rotation in 


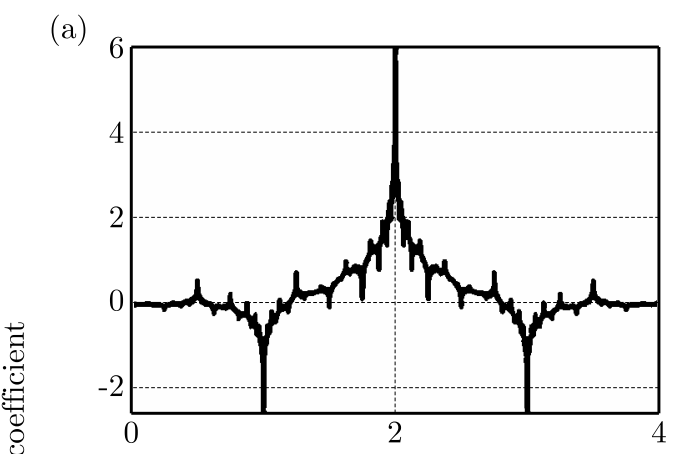

(b)
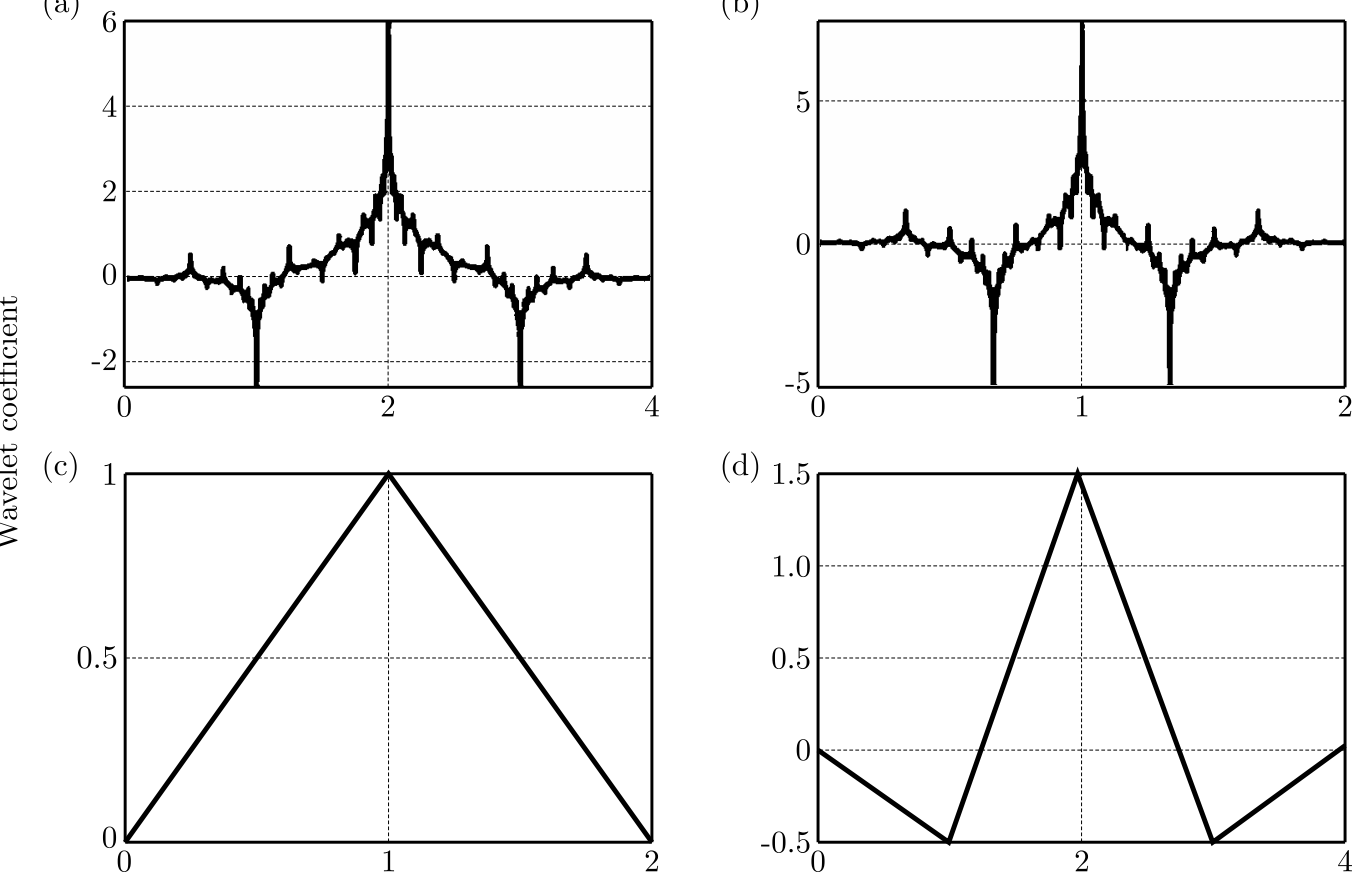

(d)

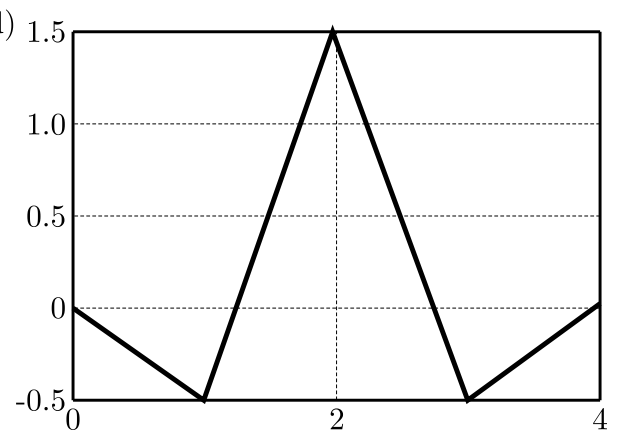

Fig. 2. Biorthogonal 2.2 wavelet: (a) analysis scaling function, (b) analysis wavelet function, (c) synthesis scaling function, (d) synthesis wavelet function

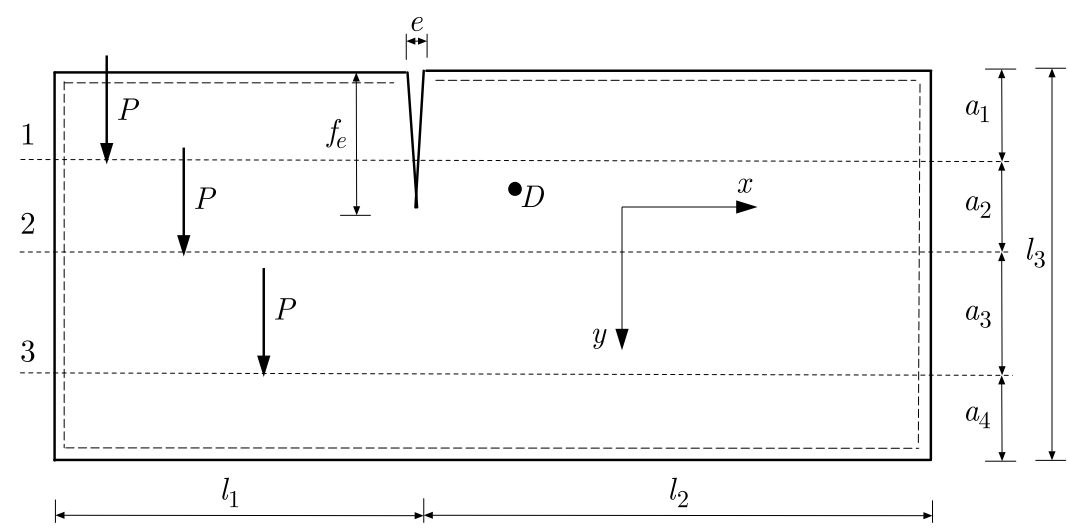

Fig. 3. Defective plate structure

arbitrary direction $\varphi$, curvatures $\kappa$ or internal forces such as bending and twisting moments or transverse forces as the response of the structure.

The measured response parameters have a character of the influence lines in its discrete form. The signal of the structural response defined in this way is processed while using DWT whose basis is described in Section 2.

The plate bending is described as the Boundary Element Method in a simplified modified approach where there is no need to introduce concentrated forces at the plate corners and equivalent shear forces at the plate continuous edges. This approach was widely described for static, dynamic and stability analysis in Guminiak (2007, 2014) and Guminiak and Sygulski (2007). The boundary integral equations are derived from Betti's theorem. For static analysis of a plate subjected by an external distributed load $q$ and concentrated force $P$, the governing equations have the form 


$$
\begin{aligned}
& c(\mathbf{x}) w(\mathbf{x})+\int_{\Gamma}\left[T_{n}^{*}(\mathbf{y}, \mathbf{x}) w(\mathbf{y})-M_{n s}^{*}(\mathbf{y}, \mathbf{x}) \frac{d w(\mathbf{y})}{d s}-M_{n}^{*}(\mathbf{y}, \mathbf{x}) \varphi_{n}(\mathbf{y})\right] d \Gamma(\mathbf{y}) \\
& =\int_{\Gamma}\left[\widetilde{T}_{n}(\mathbf{y}) w^{*}(\mathbf{y}, \mathbf{x})-M_{n}(\mathbf{y}) \varphi_{n}^{*}(\mathbf{y}, \mathbf{x})\right] d \Gamma(\mathbf{y})+\int_{\Omega} q(\mathbf{y}) w^{*}(\mathbf{y}, \mathbf{x}) d \Omega(\mathbf{y})+P(\mathrm{i}) w^{*}(\mathrm{i}, x) \\
& c(\mathbf{x}) \varphi_{n}(\mathbf{x})+\int_{\Gamma}\left[\bar{T} n^{*}(\mathbf{y}, \mathbf{x}) w(\mathbf{y})-\bar{M}_{n s}^{*}(\mathbf{y}, \mathbf{x}) \frac{d w(\mathbf{y})}{d s}-\bar{M}_{n}^{*}(\mathbf{y}, \mathbf{x}) \varphi_{n}(\mathbf{y})\right] d \Gamma(\mathbf{y}) \\
& =\int_{\Gamma}\left[\widetilde{T}_{n}(\mathbf{y}) \bar{w}^{*}(\mathbf{y}, \mathbf{x})-M_{n}(\mathbf{y}) \bar{\varphi}_{n}^{*}(\mathbf{y}, \mathbf{x})\right] d \Gamma(\mathbf{y})+\int_{\Omega} q(\mathbf{y}) \bar{w}^{*}(\mathbf{y}, \mathbf{x}) d \Omega(\mathbf{y})+P(\mathrm{i}) \bar{w}^{*}(\mathrm{i}, x)
\end{aligned}
$$

where the fundamental solution of the biharmonic equation

$$
\nabla^{4} w^{*}(\mathbf{y}, \mathbf{x})=\frac{1}{D} \delta(\mathbf{y}, \mathbf{x})
$$

is given as Green's function

$$
w^{*}(\mathbf{y}, \mathbf{x})=\frac{1}{8 \pi D} r^{2} \ln r
$$

for a thin isotropic plate, $r=|\mathbf{y}-\mathbf{x}|, \delta$ is the Dirac delta, $x$ is the source point and $y$ - field point, $D=E h^{3} /\left[12\left(1-\nu^{2}\right)\right]$ is the plate stiffness, $h$ - plate thickness, $E$ and $\nu$ are the Young's modulus and Poisson's ratio. The coefficient $c(\mathbf{x})$ is taken as $c(\mathbf{x})=1$ when $\mathbf{x}$ is located inside the plate domain; $c(\mathbf{x})=0.5$ when $\mathbf{x}$ is located on the smooth boundary and $c(\mathbf{x})=0$ when $\mathbf{x}$ is located outside the plate domain. The second boundary integral equation (2.9) can be obtained by replacing the unit concentrated force $P^{*}=1$ and the unit concentrated moment $M_{n}^{*}=1$. Such a replacement is equivalent to differentiation of the first boundary integral equation $(3.1)_{1}$ with respect to the coordinate $n$ at a point $\mathbf{x}$ belonging to the plate domain and letting this point approach the boundary and taking $n$ coincide with the normal to it. The expression $\widetilde{T}_{n}(\mathbf{y})$ denotes the shear force for clamped and for simply-supported edges, $\widetilde{T}_{n}(\mathbf{y})=V_{n}(\mathbf{y})$ (an equivalent shear force) on the boundary far from the corner in the case of a simply supported edge or $\widetilde{T}_{n}(\mathbf{y})=R_{n}(\mathbf{y})$ (distributed reaction force) on a small fragment of the boundary close to the corner. As the concentrated force at the corner is used only to satisfy the differential biharmonic equation of the thin plate, one can assume that it could be distributed along a plate edge segment close to the corner (Guminiak and Sygulski, 2007). The relation between $\varphi_{s}(\mathbf{y})$ and the deflection is specified by a simple relation $\varphi_{s}(\mathbf{y})=d w(\mathbf{y}) / d s$, hence the angle of rotation in the tangent direction $\varphi_{s}(\mathbf{y})$ can be evaluated using a finite difference scheme of the deflection with two or more adjacent nodal values. In the present analysis, the employed finite difference scheme includes the deflections of two adjacent nodes.

\section{Procedure for crack identification}

\subsection{Wavelet selection}

The fundamental question that arises before the application of wavelet analysis in any kind of time series is about the selection of the most appropriate wavelet. In our case, a preliminary selection has been made in accordance to the available selections in the Matlab Toolbox (Misiti et al., 2000) since this is the software in which the proposed method is implemented. Following the recommendations from Section 2, we have explored several biorthogonal wavelets before we concluded that the biorthogonal 2.2 wavelet performed better. 


\subsection{Proposed methodology}

The inspired idea behind the use of wavelets for crack identification lies in the fact that the existence of cracks introduces discontinuities in the structural response (Ovanesova and Suarez, 2004; Mallat, 1989; Meyer, 1992). It is not rare that these discontinuities cannot be visually observed from response signals (i.e. amplitudes of displacement), but they can be easily identified when these signals are projected onto the wavelet domain, especially from the detail coefficients. Thus, our novel approach focuses on the identification and enhancement of the information that we can derive from wavelet detail coefficients and can be directly correlated with crack location. The original procedure for crack identification can be summarized as below:

- Calculate (or measure in the case of real experiments) the signal that can be directly associated to the structural response. In the current study, we use curvatures $\kappa_{x}, \kappa_{y}$ and vertical displacement signals $w$.

- Compute the low level (up to scale 4) detail coefficients. This low level selection was successfully applied in previous studies as well (Ovanesova and Suarez, 2004).

- The crack location can be estimated as the local maximum (LM) of the absolute value in the detail coefficient.

In the case in which the aforementioned estimation is not clear (i.e. for noisy signals or when detail coefficients present more than one nearby LM) apply an appropriate estimator to isolate the LMM. In the current study, a moving variance estimator (MVE) is used as below

$$
\operatorname{MVE}=\frac{1}{n-1} \sum_{i=1}^{n}\left(x_{i}-\bar{x}_{i}\right)^{2}
$$

where $n$ is number of samples in the moving window. Small values of $n$ provide a more rapid detection of sharp changes (increased sensitivity) but may lead to false estimations due to outliers (decreased selectivity). On the contrary, higher values of $n$ are not prone to sharp peaks or outliers but they introduce significant delays between the real and the detected peak. In our case, $n=3$ is selected as the best compromise between sensitivity and selectivity. This value corresponds to a moving window with length around $5 \%$ of the total number of measurements (which are $N=64$ ).

The LM from detail coefficients are projected as the maximum of the moving variance estimator.

\section{Numerical results}

The aim of this work is to detect localization of a defect provided that the damage (crack) exists in the considered plate structure. Numerical investigation is conducted basing on signal analysis of the structural static response. A rectangular plate structure, simply-supported on the boundary is considered. The Boundary Element Method is applied to solve a thin plate bending problem. Each plate edge is divided into 30 boundary elements of the constant type. The collocation point is located slightly outside the plate edge, which is estimated by parameter $\varepsilon=\delta / d$, where $\delta$ is the real distance of the collocation point from the plate edge, and $d$ is the element length (Guminiak, 2014). For each example, $\varepsilon=0.001$ is assumed. The diagonal boundary terms in the characteristic matrix are calculated analytically, and the rest of them using 12-point Gauss quadrature. The plates properties are: $E=205.0 \mathrm{GPa}, \nu=0.3, h=0.02 \mathrm{~m}$. All plates are loaded statically. A static concentrated external load $P=1000 \mathrm{~N}$ is replaced by an equivalent constant distributed loading $q$ acting over the square surface of dimensions $0.05 \mathrm{~m} \times 0.05 \mathrm{~m}$. Plate defects are introduced by the additional boundaries (free edges) forming 
a hole in relation to the basic plate domain. The static concentrated load is applied at selected points along the direction parallel to one plate dimension. As the structural response, deflections and curvatures are taken into account. Curvatures $\kappa_{x}, \kappa_{y}$ can be identified using displacements given in three points: left, central and right, where the left and right points are located in direct vicinity of the central point. The curvature $\kappa_{x y}$ can be found while using displacements of four points located in direct vicinity of the central point. Subsequently, the curvatures can be calculated using classic difference operators. The measurement point is located near the damaged area of the considered plate. The minimum number of measurements is equal to 32 (Knitter-Piątkowska et al., 2014). In the considered examples, a number of 64 measurements has been applied.

\subsection{Plate with middle crack}

The plate loaded by a static concentrated $P$ force is considered and presented in Fig. 4 . The coordinates of the measurement point $A$ are: $x_{A}=2.15 \mathrm{~m}$ and $y_{A}=0.25 \mathrm{~m}$. The introduced plate defect is described by the parameter $e=0.005 \mathrm{~m}$.

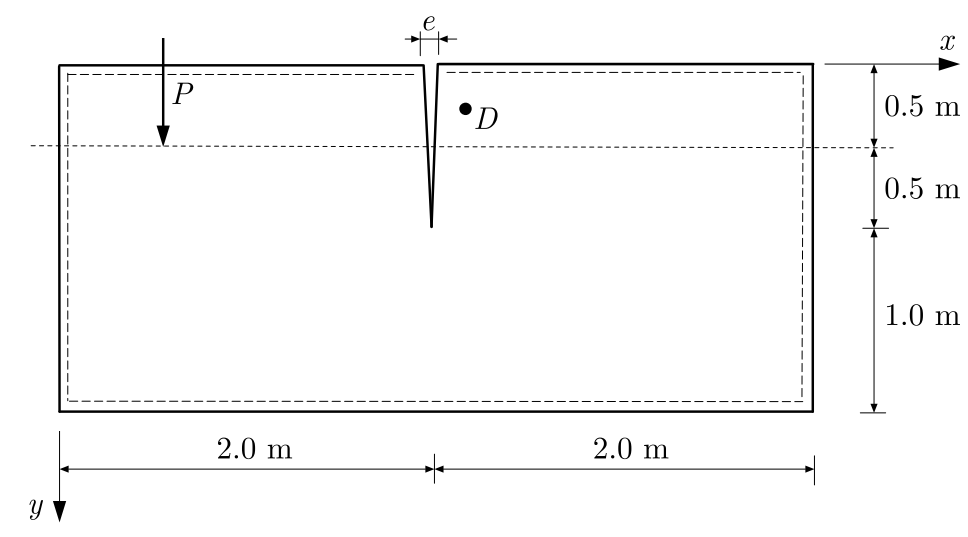

Fig. 4. The considered plate structure with a middle crack

The response signals that are used for the crack identification are: curvature $\kappa_{x}$, curvature $\kappa_{y}$ and displacement $w$, which are presented in Fig. 5, respectively.

For each above signal, a DWT using bior.2.2 wavelet has been performed. The obtained results are shown in Fig. 6 for detail 1 coefficients. The detail coefficients from the remaining scales of each transform are not presented since the significant information can be derived from coefficients of scale 1 only. At each figure, location of the crack is depicted as a vertical dotted line. A common pattern is unfolded for all three signals: the LMM clearly indicates the crack position. More specificly, for signal $\kappa_{x}$, the crack position is identified from the maximum of detail 1 coefficient while for $\kappa_{y}$ and displacement signals, the crack position is identified from the minimum. The importance of the previous observation is based on the fact that a simple threshold detector (applied to detail 1 coefficients) is used, and as a result, we can identify location of the crack with a significant accuracy.

A criticism to the previous findings can be raised if we assume that one of the response signals $\kappa_{y}$ (and probably other signals that are contaminated by noise), presents nearby LMM (in form of subordinate peaks) that may affect the accuracy of threshold detection that we propose earlier. For this reason, we applied MVE to detail 1 coefficients in order to enhance information of the crack location and thus to enhance the accuracy of the proposed threshold detector. Figure 7 shows the results where the crack location is presented with a very clear peak of MVE. This event eliminates any uncertainty about the crack identification procedure and provides a clear answer to the question "if there is a crack and where it is located". 
(a)

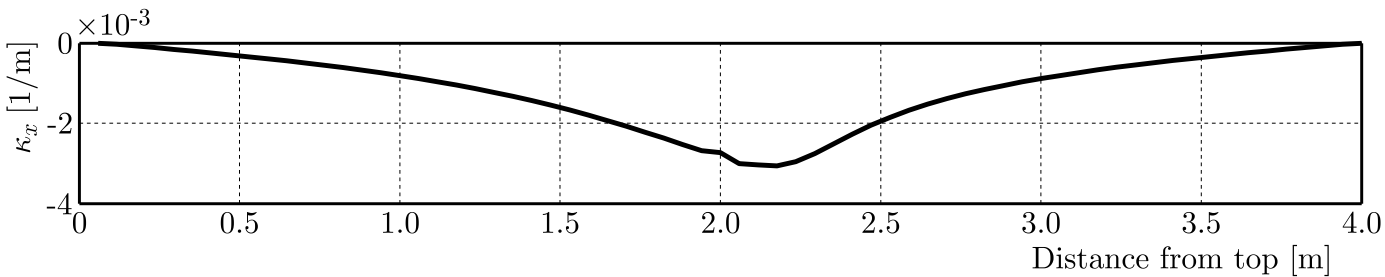

(b)

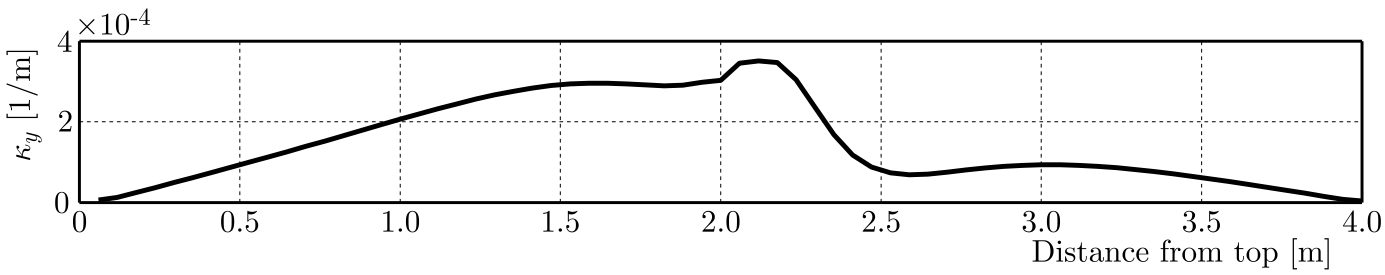

(c)

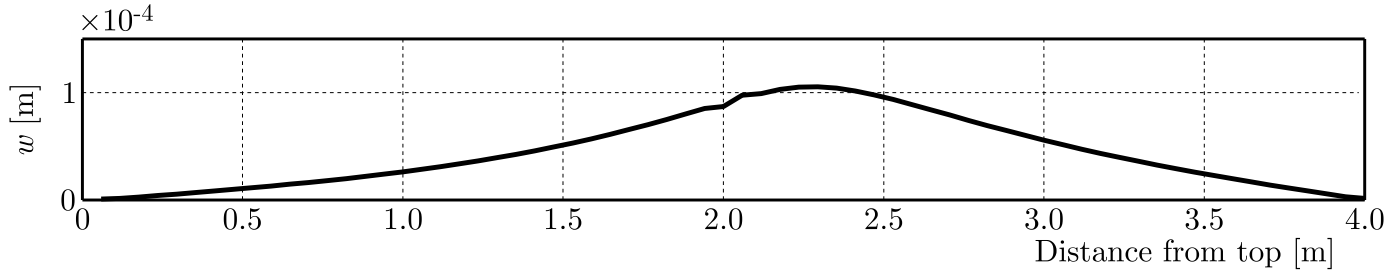

Fig. 5. Response signals for the plate with a middle crack: (a) curvature $\kappa_{x}$, (b) curvature $\kappa_{y}$ and (c) displacement $w$; number of measurements $N=64$

(a)

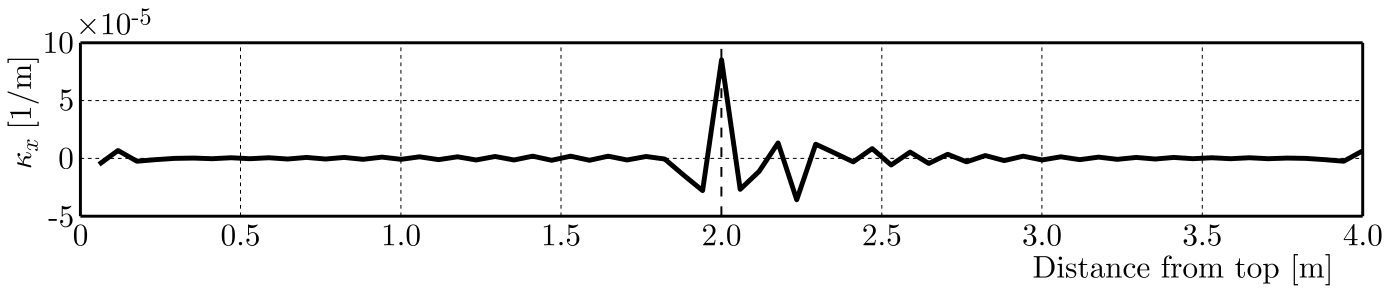

(b)

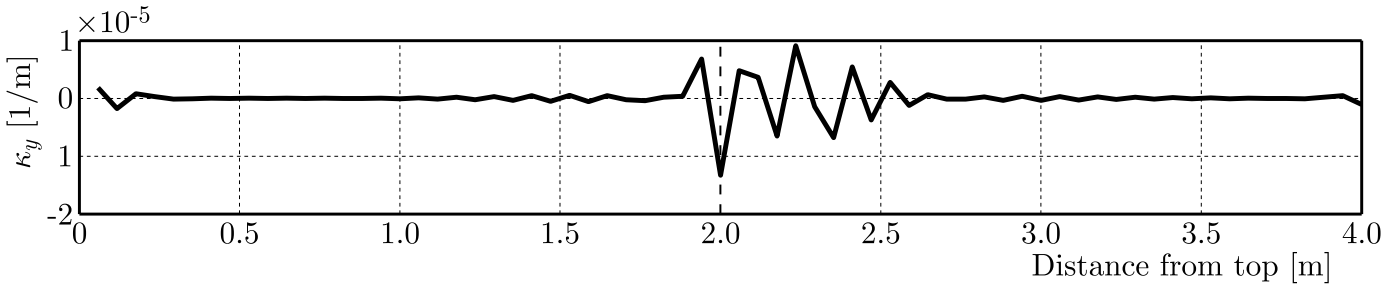

(c)

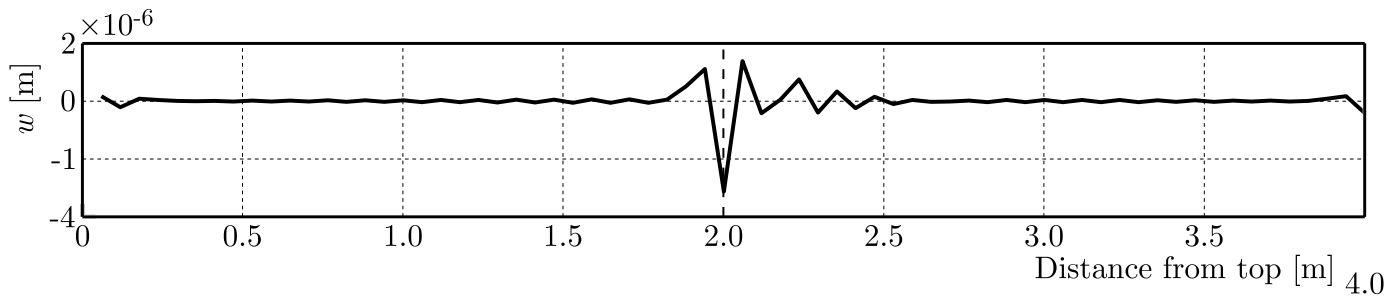

Fig. 6. Detail coefficients at scale 1 of (a) curvature $\kappa_{x}$, (b) curvature $\kappa_{y}$ and (c) displacement signals $w$ of the plate with a middle crack; vertical dotted line indicates the actual location of the crack

\subsection{Plate with a corner crack}

The plate loaded by a static concentrated $P$ force is considered and presented in Fig. 8 . The coordinates of the measurement point $D$ are: $x_{A}=3.75 \mathrm{~m}$ and $y_{A}=0.35 \mathrm{~m}$. The introduced plate defect is described by parameters: $e_{1}=e_{2}=e_{3}=0.005 \mathrm{~m}$.

The same procedure as in Section 5.1 is applied to the plate with a corner crack. Figure 9 shows the response signals where the corresponding results from DWT are shown in Fig. 10. 
(a)

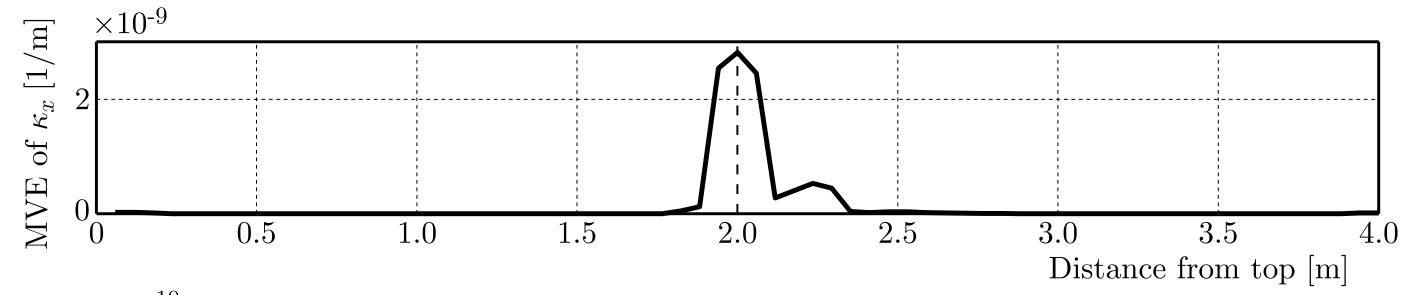

(b)

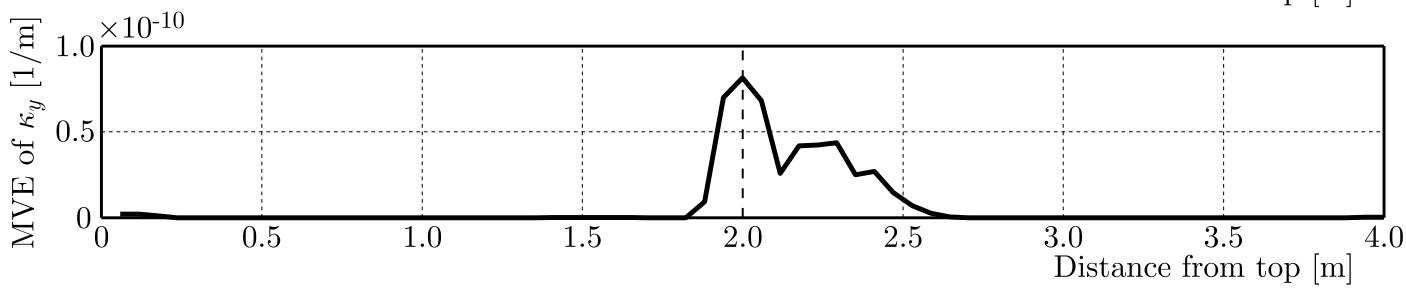

(c)

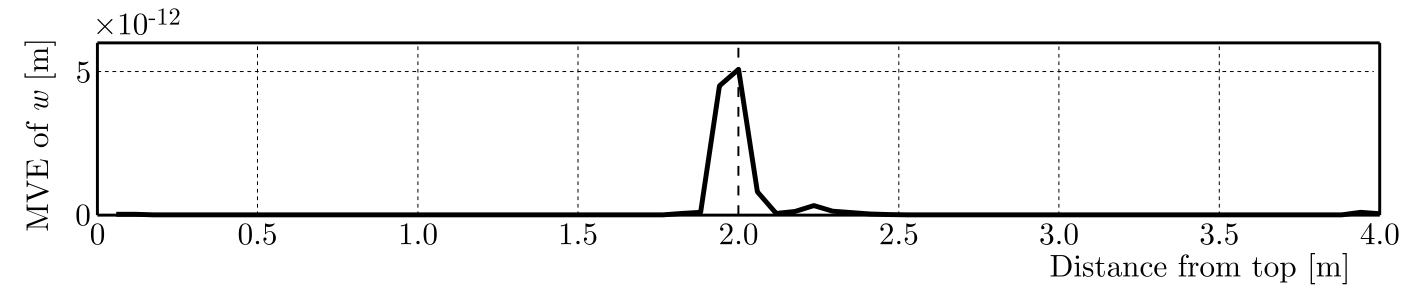

Fig. 7. MVE estimations for detail 1 coefficients of (a) curvature $\kappa_{x}$, (b) curvature $\kappa_{y}$ and

(c) displacement signals $w$ of the plate with a middle crack; vertical dotted line indicates the actual location of the crack

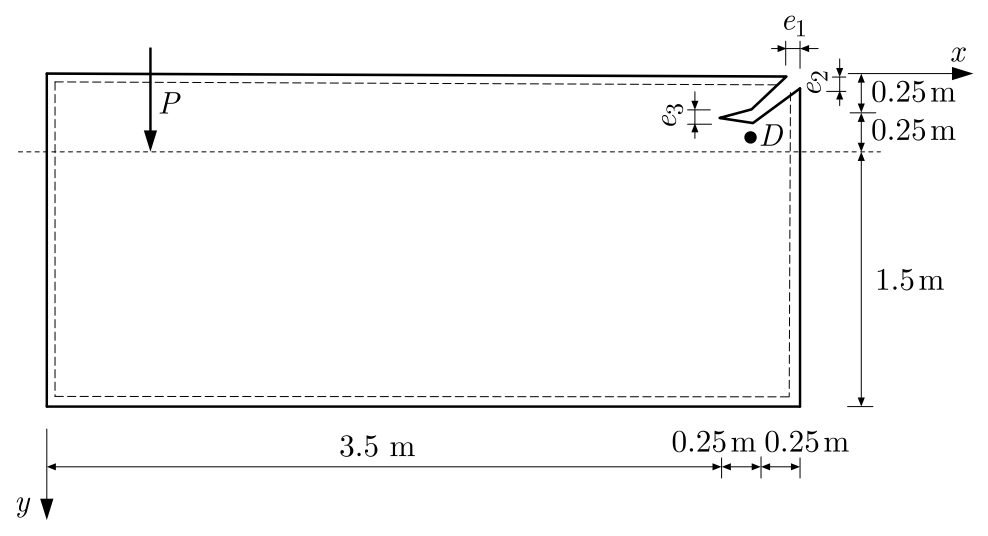

Fig. 8. Considered plate structure with a corner crack

The used wavelet is the bior.2.2, and the detail coefficients at scale 4 are taken into account. The vertical dotted line indicates the beginning of the corner crack. The results are clearer than in the case of the plate with the middle crack: the LMM can be easily detected as there are no subordinate peaks in the detail coefficients. The LMM again successfully points out the location of the crack. Thus, the use of MVE in this case is not presented since it will not add significant information to the crack identification procedure.

An important note, common for both cases, is regarding the detail coefficients amplitudes. As we can observe, the amplitude of detail coefficients is 50 times smaller than the amplitude or the corresponding response signal. This, in turn, means that the details coefficients are prone to add with white noise. The level of noise influence is further examined in the next Section.

\subsection{Noise immunity test}

Due to unavailability of experimental data, the proposed method has been tested for its noise immunity by adding to the response signals independent realizations of artificial White Gaussian 
(a)

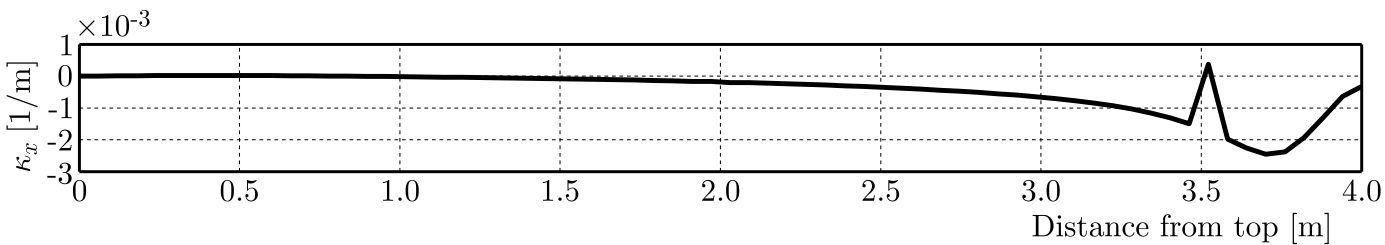

(b)

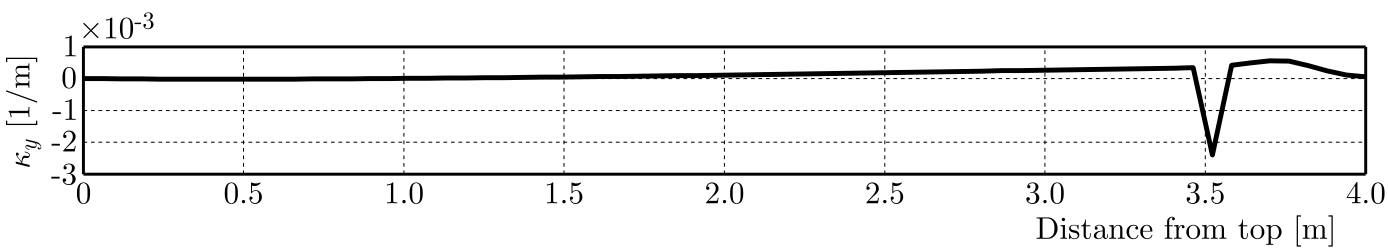

(c)

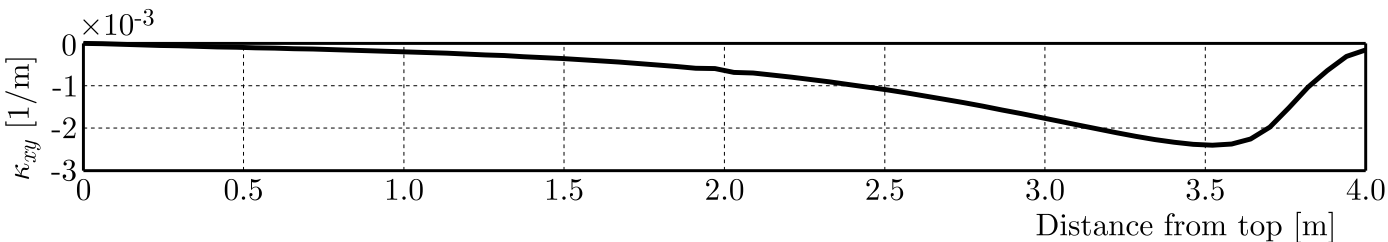

(d)

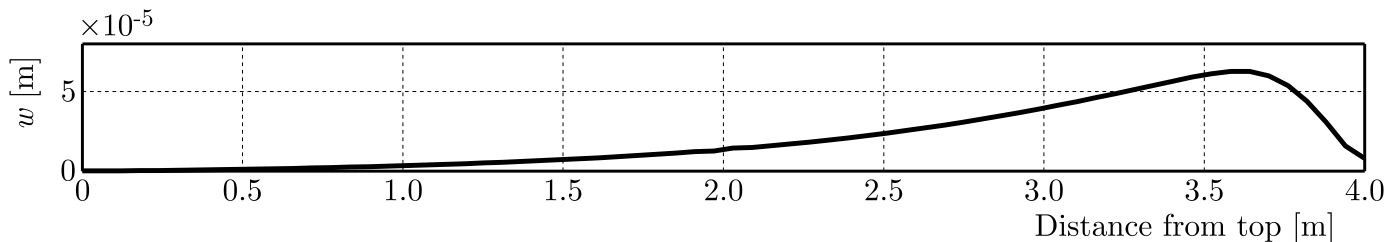

Fig. 9. Response signals for the plate with a corner crack (a) curvature $\kappa_{x}$, (b) curvature $\kappa_{y}$, (c) curvature $\kappa_{x y}$, and displacement $w$

(a)

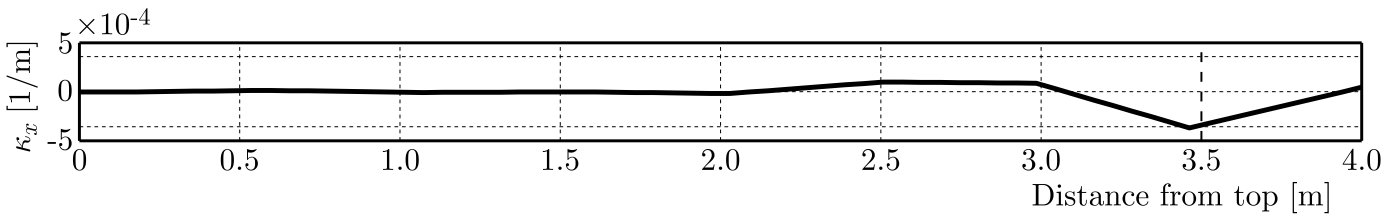

(b)

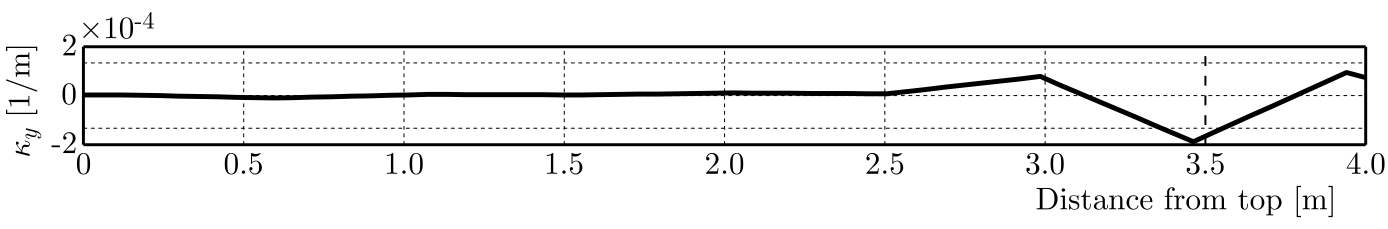

(c)

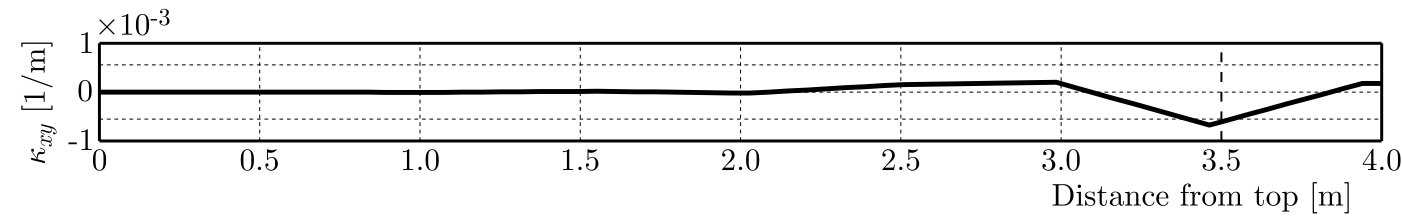

(d)

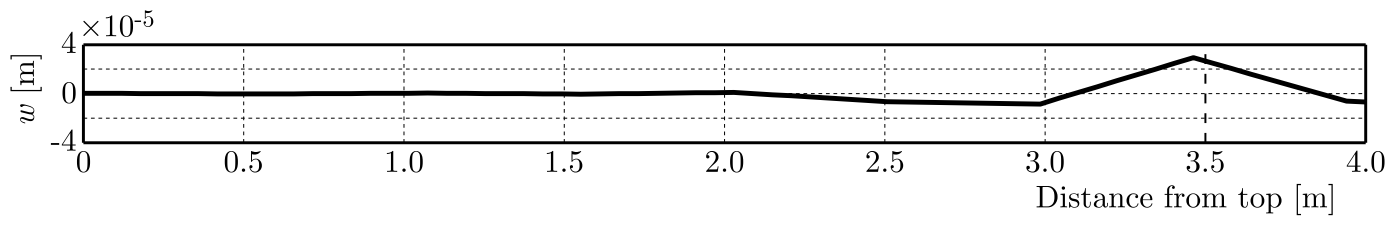

Fig. 10. Detail coefficients at scale 4 of: (a) curvature $\kappa_{x}$, (b) curvature $\kappa_{y}$, (c) curvature $\kappa_{x y}$ and (d) displacement $w$ signals of plate with corner crack; vertical dotted line indicates the actual location of crack 
Noise (WGN). To ensure a large scale assessment, a range of 81 Signal-to-Noise (SNR) cases are considered (for each plate) in order to represent several measurement conditions. The range of the examined signals begins from $100 \mathrm{db}$ (almost noiseless) up to $20 \mathrm{db}$ (quite noisy signal) in $1 \mathrm{db}$ decrements. Figure 11 presents the latter case $(\mathrm{SNR}=20 \mathrm{db})$ for signals from the plate with a middle crack, whereas in Fig. 12, the corresponding MVE results are presented.

(a)

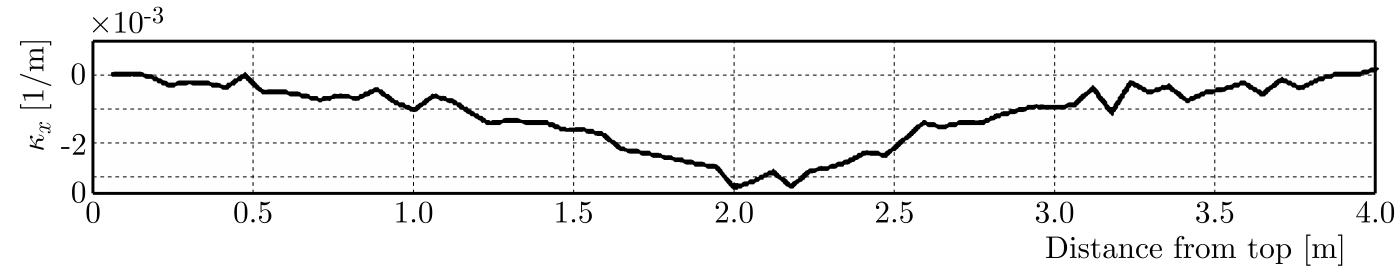

(b)

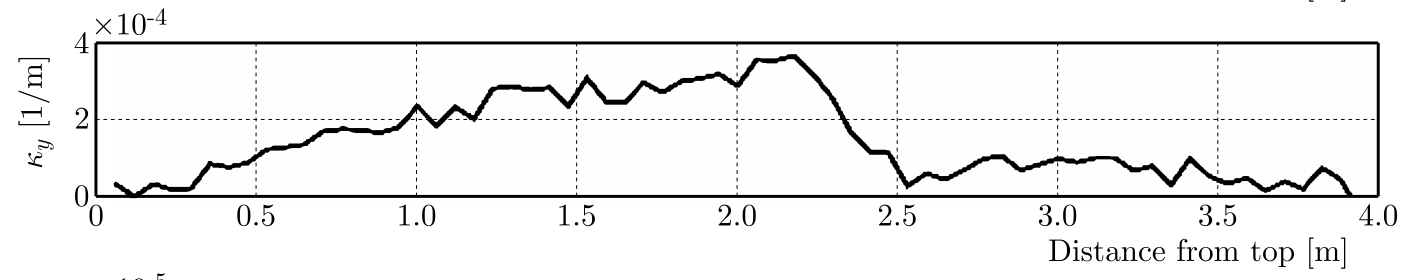

(c)

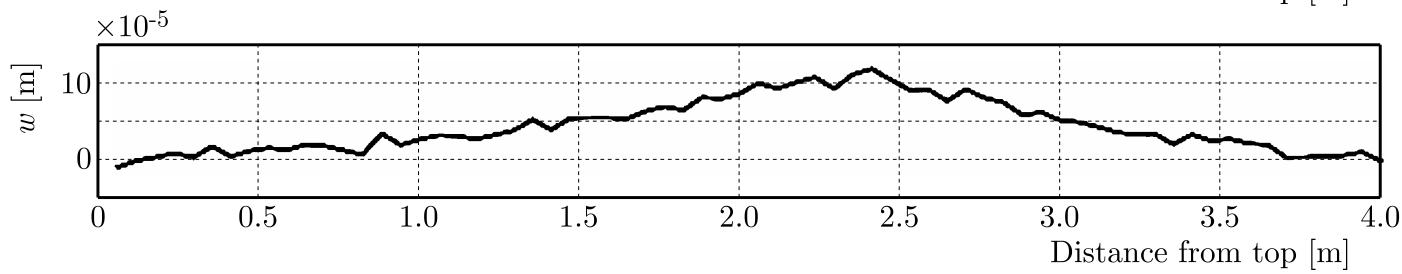

Fig. 11. The noisy case $(\mathrm{SNR}=20 \mathrm{db})$ of response signals for the plate with a middle crack:

(a) curvature $\kappa_{x}$, (b) curvature $\kappa_{y}$ and (c) displacement $w$
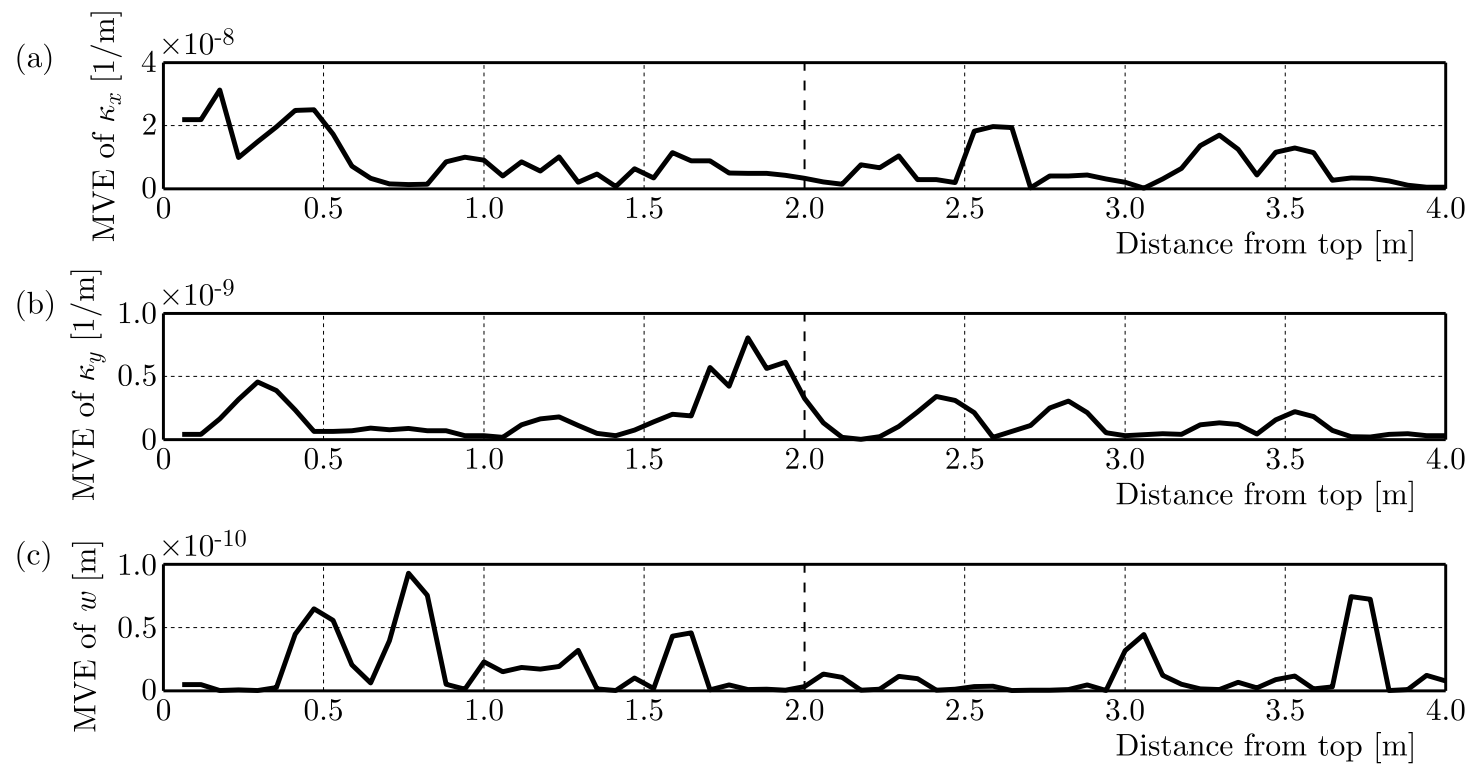

Fig. 12. MVE estimations for detail 1 coefficients of (a) curvature $\kappa_{x}$, (b) curvature $\kappa_{y}$ and

(c) displacement signals $w$ with $\mathrm{SNR}=20 \mathrm{db}$ of the plate with a middle crack; vertical dotted line indicates the actual location of the crack

From Fig. 12, it is obvious that MVE fails to detect the crack location for every noisy signal. This situation is observed in the plate with the corner crack as well. Such observations have led us to the examination of the minimum $\mathrm{SNR}_{\min }$ where the proposed method can successfully estimate the crack location. For example, with signals having SNR $=45 \mathrm{db}$, it is still possible to 
identify the location as presented in Fig. 13. The results from the aforementioned noise tests are shown in Fig. 14. The results from Fig. 14 indicate that there is no common $\mathrm{SNR}_{\text {min }}$, but it is noticeable that for SNR $\geqslant 40 \mathrm{db}$ the proposed method can estimate the crack location accurately. In addition, the results from noise tests for the plate with the corner crack reveal that the performance of MVE estimations for $\kappa_{x}$ and $\kappa_{y}$ signals is quite remarkable. This observation must be expected since (as shown in Fig. 9) these two specific signals present intrinsic discontinuity at the crack location (thus the DWT can easily detect it). The results from the curvature $\kappa_{x y}$ and displacement $w$ signals are less prominent but their $\mathrm{SNR}_{\text {min }}$ remains below $40 \mathrm{db}$. The detailed results of the performance of MVE on each signal can be found in Table 1.

(a)

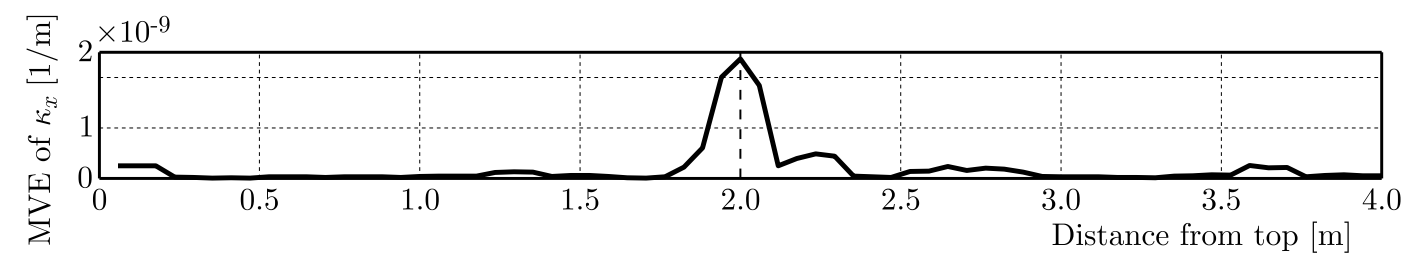

(b)

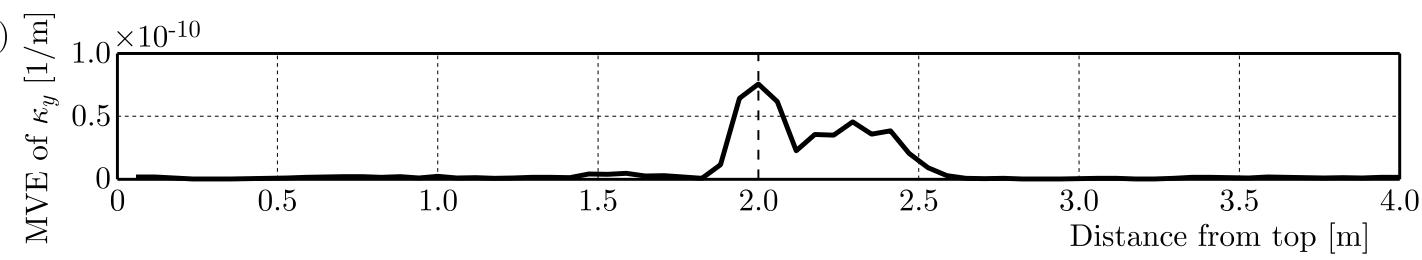

(c)

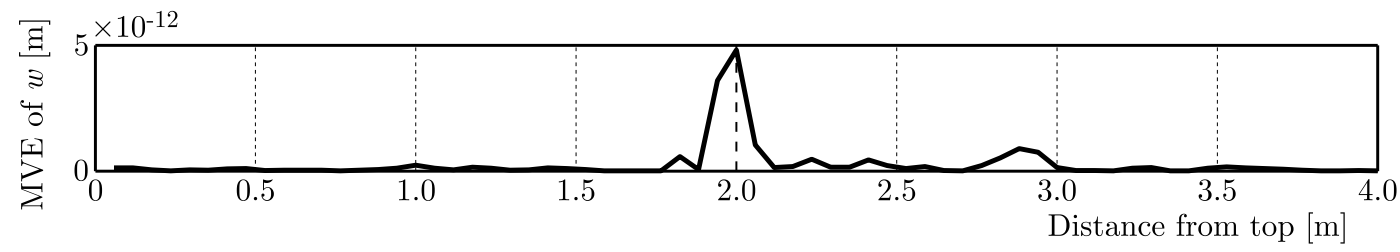

Fig. 13. MVE estimations for detail 1 coefficients of (a) curvature $\kappa_{x}$, (b) curvature $\kappa_{y}$ and (c) displacement signals $w$ with $\mathrm{SNR}=45 \mathrm{db}$ of the plate with a middle crack; vertical dotted line indicates the actual location of the crack

(a)

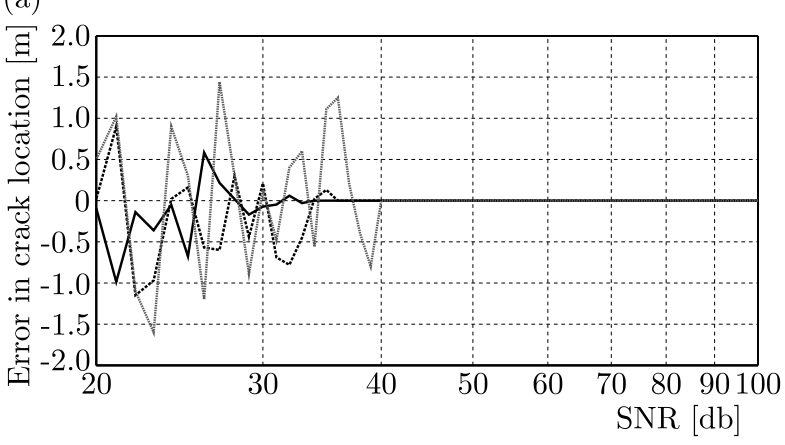

(b)

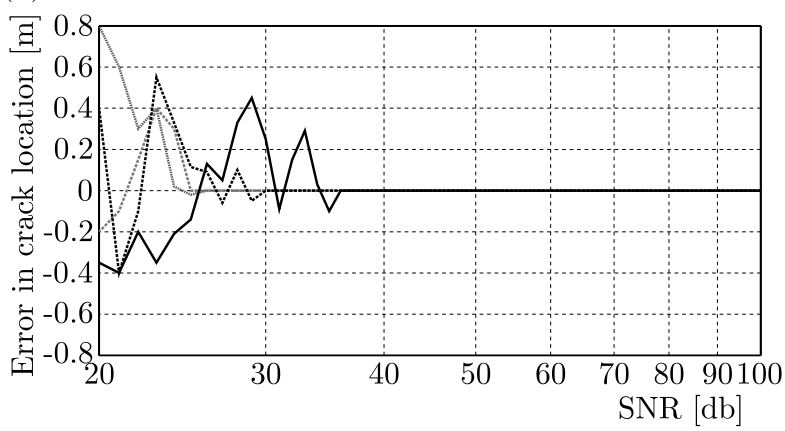

Fig. 14. MVE errors in crack location estimation for response signals with the middle crack (a) and the corner crack (b). The SNR is ranging from $20 \mathrm{db}$ to $100 \mathrm{db}$ (in $1 \mathrm{db}$ steps): curvature $\kappa_{x}$

(black solid line), curvature $\kappa_{y}$ (black dashed line), displacement $w$ (grey dotted line) and curvature $\kappa_{x y}$ (grey dashed line)

Stacking the previous results together, it is clear that the crack location identification will be influenced (at least in one case) by noise if the corresponding response signals have SNR $<40 \mathrm{db}$. This is the conservative point of view since there are response signals (the $\kappa_{x}$ and $\kappa_{y}$ ) that can perform nearly perfect (i.e. exact identification of crack location) with $\mathrm{SNR}_{\text {min }}$ reduced down to $36 \mathrm{db}$. The previous observations dictate that the proposed method is quite robust to additive 
Table 1. Noise immunity test results

\begin{tabular}{|l|c|c|c|c|c|c|c|}
\cline { 2 - 8 } \multicolumn{1}{c|}{} & \multicolumn{3}{c|}{ Plate with middle crack } & \multicolumn{4}{c|}{ Plate with corner crack } \\
\hline response signal & $\kappa_{x}$ & $\kappa_{y}$ & $w$ & $\kappa_{x}$ & $\kappa_{y}$ & $\kappa_{x y}$ & $w$ \\
\hline \hline $\mathrm{SNR}_{\min }[\mathrm{db}]$ & 34 & 36 & 40 & 25 & 26 & 30 & 36 \\
\hline
\end{tabular}

noise, and for this reason, further study with experimental data must take place in order to validate the above conclusions.

\section{Conclusions}

The implementation of DWT for identification of signal discontinuity in the analysis of plate structures is presented in the given paper. Bending of a thin plate (static analysis) is described by boundary integral equations and solved using the BEM. Despite the fact, the studied problem is two-dimensional from the point of view of deformation description. Application of one-dimensional discrete DWT leads to satisfying results in defect detection. The proposed approach allows discovering small disturbances in the response signal of a defective structure and does not require a reference to the signal from an undamaged structure (additional errors avoided). The considered examples proved that DWT of the structural response signal expressed in deflections or curvatures established at a selected domain point, quite correctly identifies the presence and position of the defect. The data are gathered in one measurement point in equal time intervals. The detection of defect position can easily be implemented by using a simple threshold detector applied to the corresponding DWT detail coefficients. In the case where a small uncertainty exists, the making use of an additional detector (called Moving Variance Estimator MVE) is proposed. The purpose of MvE is to enhance the information that may remain "hidden" (due to non-distinguishable peak) or "blurred" (due to subsequent peaks) near defect position, and this is the original element in the paper. The results indicate that the MVE performs quite satisfactory on deriving accurately the position of the defect, especially for deformation response signals.

Since the current study is a numerical one, it is expected that there will be a contamination of response signals by noise in real experiments. For this reason, additional research regarding the performance of the proposed method over noisy signals has been carried out. More specificly, we add independent realizations of artificial White Gaussian Noise to response signals generating a dataset with SNR from 20db to 100db (in 1db increments). We have noticed that even with the presence of noise there is a minimum SNR over which it is possible to reveal accurately the position of the defect using MVE. This minimum SNR has been estimated for both plates and for every response signal.

The importance and novelty of the current study does not lie only in the application of DWT for crack identification but goes a step further to the implementation of a real time system for crack location. In a such way, a prototype system will be highly benefited from the use of DWT since it is computationally efficient regarding signal processing resources. Moreover, by using the lifting approach implementation algorithm (Daubechies and Sewldens, 1998) we will be able to adapt two main advantages of the lifting approach against the widely used polyphase algorithm: doubling computation speed (Sweldens, 1996) and in-place computation of coefficients (without allocating extra memory) (Meyer, 1992). The above properties may be mission-critical in realworld installations where small memory buffers and very low-power microprocessors perform necessary calculations in wireless sensor network implementation. 


\section{References}

1. Burczyński T., Kuś W., DŁugosz A., Orantek P., 2004, Optimization and defect identification using distributed evolutionary algorithms, Engeinnering Applications of Artificial Intelligence, 17, 337-344

2. Chang C., Chen L., 2004, Damage detection of a rectangular plate by spatial wavelet based approach, Applied Acoustic, 65, 819-832

3. Cohen A., Daubechies J., Feauveau J., 1992, Biorthogonal bases of compactly supported wavelets, Communications on Pure and Applied Mathematics, 45, 485-560

4. Daubechies I., 1992, Ten Lectures on Wavelets, Philadelphia: Society for Industrial and Applied Mathematics

5. Daubechies I., Sweldens, W., 1998, Factoring wavelet transforms into liftin steps, Journal of Fourier Analysis and Applications, 4, 3

6. Dems K., Mróz, Z., 2001, Identification of damage in beam and plate structures using parameterdependent frequency changes, Engineering Computations, 18, 1/2, 96-120

7. Douka E., Loutridis S., Trochidis A., 2003, Crack identification in beams using wavelet analysis, International Journal of Solid and Structures, 40, 3557-3569

8. Douka E., Loutridis S., Trochidis A., 2004, Crack identification in plates using wavelet analysis, Journal of Sound and Vibration, 270, 279-295

9. Garstecki A., Knitter-Piatkowska A., Pozorski Z., Ziopaja K., 2004, Damage detection using parameter dependent dynamic experiments and wavelet transformation, Journal of Engineering and Management, 10, 3, 191-197

10. Gentile A., Messina A., 2003, On the continuous wavelet transforms applied to discrete vibrational data for detecting open cracks in damaged beams, International Journal of Solid and Structures, 40, 295-315

11. Gros X., 1995, An eddy current approach to the detection of damage caused by low-energy impacts on carbon fiber reinforced materials, Meterials and Design, 16, 3, 167-173

12. Guminiak M., 2007, Static analysis of thin plates by the boundary Element Method in a nonsingular approach, Foundations of Civil and Environmental Engineering, 9, 75-93

13. Guminiak M., 2014, An alternative approach of initial instability analysis of Kirchhoff plates by the Boundary Element Method, Engineering Trasnactions, 62, 1, 33-59

14. Guminiak M., Sygulski R., 2007, Vibrations of plates immersed in compressible fluid by the BEM, 9th International Conference on Modern Building Materials, Structures and Techniques, P.V.M.J. Skibniewski (Edit.), Vilnius, Vilnius Gediminas Technical University Press "Technika", 1453, 925-930

15. Jensen A., La Cour-Harbo A., 2001, Ripplies in Mathematics, Berlin, Springer

16. Kim H., Melhem H., 2004, Damage detection in structures by wavelet analysis, Engineering Structures, 26, 347-362

17. Knitter-Piatkowska A., Garbowski, T., 2013, Damage detection through wavelet transform and inverse analysis, VI International Conference on Adaptive Modelling and Simulation (ADMOS 2013), J. Moitinho de Almeida, P. Diez, C. Tiago, and N. Pares (Edit.), Barcelona, 389-400

18. Knitter-Piątkowska A., Guminiak M., Przychodzki M., 2014, Damage Detection in Truss Structure Being the Part of Historic Railway Viaduct Wavelet Transformation, T. Eodygowski, J. Rakowski and P. Litewka (Edit.), CRC Press/Balkema, Taylor and Francis Group

19. Lee J., Seo D.-W., Shoji T., 2004, Numerical consideration of magnetic camera for quantitative nondestructive evaluation, Key Engineering Materials, 270-273, 630-635

20. LIEW K., WANQ Q., 1998, Application of wavelet theory for crack identification in structures, Journal of Engineering Mechanics, 124, 2, 152-157 
21. Loutridis S., Douka E., Hadjileontiadis L., Trochidis A., 2005, A two-dimensional wavelet transform for detection of cracks in plates, Engineering Structures, 27, 1327-1338

22. Mallat S., 1989, A theory for multiresolution signal decomposition: the wavelet representation, IEEE Transactions on Pattern Analysis and Machine Intelligence, 11, 7, 674-693

23. Meyer Y., 1992, Wavelets and Operators. Cambridge Studies in Advanced Mathematics, Cambridge, UK, Cambridge University Press

24. Misiti M., Misiti Y., Oppenheim G., Poggi J.M., 2000, Wavelet Toolbox - User's Guide, Natick, MA, The MathWorks

25. Ostachowicz W., KaczmarczyK S., 2001, Vibrations of composite plates with SMA fibres in a gas stream with defects of the type of delamination, Composite Structures, 54, 305-311

26. Ovanesova A., Suarez L., 2004, Applications of wavelet transforms to damage detection in frame structures, Engineering Structures, 26, 39-49

27. Quek S., Wanq Q., Zhang L., Ang K., 2001, Sensitivity analysis of crack detection in beams by wavelet technique, International Journal of Mechanical Science, 43, 2899-2910

28. Rogers L., 2005, Crack detection using acoustic emission methods - fundamentals and applications, Key Engineering Materials, 293-294, 33-48

29. RuckA M., Wilde K., 2004, Numerical simulation of damage detection in rectangular plate by two-dimensional wavelet transform, Proceedings of International Workshop on Simulations in Urban Engineering, Gdańsk, Poland, 205-208

30. Shinoba K., Morotomi R., Mukai K., Yoshiara T., Shirai M., Miyamoto H., 2004, Application of digital radiography to aerospace-craft, Key Engineering Materials, 270-273, 1361-1365

31. Sweldens W., 1996, The lifting scheme: A custom-design construction of biorthogonal wavelets, Applied and Computational Harmonic Analysis, 3, 2, 186-200

32. WANG Q., Deng X., 1999, Damage detection with spatial wavelets, International Journal of Solids and Structures, 36, 3443-3448

33. WAsZcZyszyn Z., Ziemiański L., 2001, Neural networks in mechanics of structures and materials - new results and prospects of applications, Computers and Structures, 79, 2261-2276

34. Zhang G., Hu H., TA D., 2004, Ultrasonic detection of the metallurgical defects in the steel and its evaluation by neural networks based on the wavelet transform noise suppression, Key Engineering Materials, 270-273, 160-167

35. Ziopaja K., Pozorski Z., Garstecki A., 2011, Damage detection using thermal experiments and wavelet transformation, Inverse Problems in Science and Engineering, 19, 1, 127-153 